\title{
Influencia de los radios en algunas propiedades físicas y mecánicas de la madera de ocho encinos (Quercus) de Durango, México
}

\author{
Carmen de la Paz Pérez Olvera ${ }^{1}$ \\ Raymundo Dávalos Sotelo \\ Paz Alejandra Quintanar Isaías ${ }^{1}$
}

\begin{abstract}
RESUMEN
Se presenta la relación del parénquima radial (radios uniseriados y multiseriados) con la contracción volumétrica y las propiedades mecánicas de flexión, compresión paralela y perpendicular y el cortante paralelo de la madera de ocho especies de encinos de Durango, México. Para determinar la relación de los radios con las propiedades tecnológicas, se hizo un análisis de regresión multivariado en donde las variables dependientes fueron los valores anatómicos y las independientes los valores físicos y mecánicos. Los resultados muestran que la altura y anchura de los radios multiseriados son los caracteres anatómicos de mayor influencia en las propiedades tecnológicas. Se resalta que en la selección de la madera de encino para su aprovechamiento, se deben considerar las características anatómicas, físicas y mecánicas, que influyen en su manejo para aprovechar de manera óptima este recurso y lograr la conservación de los encinares y preservar el equilibrio ecológico de los sitios donde crecen.
\end{abstract}

PALABRAS CLAVE:

Contracción volumétrica, Durango, encino, madera, México, propiedades mecánicas, radios.

\begin{abstract}
The relations between the radial parenchyma (uniseriate and multiseriate rays) and volumetric shrinkage and with the mechanical properties of eight Durango woods are presented. The mechanical properties studied are: static bending, compression parallel-to-grain, compression perpendicular-to-grain and shear parallel-to-grain. The relationship between the anatomical and physical properties was determined through stepwise regression analysis. The dependent variables were the anatomical values and the independent ones were the physical and mechanical values. It was determined that the height and width of multiseriate rays are the anatomical characters with the greatest influence in all properties. It was concluded that the selection of oak wood species in the transformation processes is an urgent task and that the anatomical and physical characteristics that influence its management must be considered in order to utilize this resource in an optimal way and to be able to achieve the conservation of oak forests and the preservation of the ecological equilibrium of the places where they grow.
\end{abstract}

KEYWORDS:

Volumetric shrinkage, Durango, oak, wood, Mexico, mechanical properties, Rays.

1 Universidad Autónoma Metropolitana Iztapalapa. División de Ciencias Biológicas y de la Salud. Departamento de Biología. Av. San Rafael Atlixco \# 186, Col. Vicentina, Iztapalapa, D.F. C.P. 09340. México, D.F.

Tel: (55) 58044699 fax (55) 58044688 c.e.cppo@xanum.uam.mx c.e. aqi@xanum.uam.mx

2 Instituto de Ecología, A. C. Unidad de Recursos Forestales. Km 2.5 Carretera antigua a Coatepec No. 351. C.P.91070.Xalapa, Veracruz.c.e: davalos@ecología.edu.mx 


\section{INTRODUCCIÓN}

El estado de Durango está definido por tres provincias fisiográficas: Sierra Madre Occidental, Llanuras del Norte y Mesa del Centro. La Sierra Occidental abarca gran parte de su territorio, con una anchura de hasta 160 kilómetros y cumbres de más de 3000 metros de altura. En las Llanuras del Norte y del Centro se encuentran también varias sierras. Durango tiene una superficie total de $119684 \mathrm{Km}^{2}$, ocupando el cuarto lugar en extensión territorial del país. Su altitud promedio es de $1775 \mathrm{msnm}$. Las actividades económicas más importantes son la agricultura, la ganadería, la minería y la explotación forestal (SPP, 1996).

Dada su fisiografía, se presentan diferentes tipos de vegetación, entre los que se encuentran los bosques, ocupando el $46,56 \%$ de la superficie estatal, principalmente en la Sierra Madre, en donde vegetan 36 especies de Quercus: 18 encinos rojos (subgénero Erythrobalanus) y 18 encinos blancos (subgénero Leucobalanus) (Zavala 1995) (Tabla 1). Estas especies presentan diferencias tanto en sus características anatómicas como en sus propiedades tecnológicas principalmente entre individuos de los dos subgéneros (de la Paz Pérez 1974, 2000). Las especies mejor conformadas, Quercus candicans, $Q$. coccolobifolia, Q. crassifolia, Q. durifolia, Q. sideroxyla (encinos rojos), Q. convallata, $Q$. laeta y $Q$. potosina (encinos blancos), se seleccionaron para hacer la relación del parénquima radial (radios uniseriados y multiseriados) con algunas propiedades físicas y mecánicas.

De acuerdo con Kollman y Coté, 1968; Tsoumis, 1969; Jane, 1970; Panshin y de Zeeuw, 1970; Desch, 1974; Wangaard, 1981; Echenique y Robles, 1993; entre muchos autores, las propiedades de la madera son el reflejo de su estructura interna, por lo que el deseo de los estudiosos de la madera es poder predecir su comportamiento a partir de las características anatómicas, que en

Tabla 1. Especies de Quercus de Durango (Zavala, 1995)

\begin{tabular}{llll}
\hline \multicolumn{1}{c}{ Erythrobalanus (encinos rojos) } & \multicolumn{1}{c}{ Leucobalanus (encinos blancos) } \\
\hline Q. aristata & Q. gentry & Q. arizonica & Q. microphylla \\
Q. candicans & Q. hypoleucoides & Q. cordifolia & Q. oblongifolia \\
Q. castanea & Q. laurina & Q. convallata & Q. obtusata \\
Q. coccolobifolia & Q. planipocula & Q. chihuahuensis & Q. potosina \\
Q. crassifolia & Q. scytophylla & Q. excelsa & Q. praeco \\
Q. durifolia & Q. sideroxyla & Q. gregii & Q. resinosa \\
Q. eduardii & Q. urbanii & Q. grisea & Q. rugosa \\
Q. elliptica & Q. uxoris & Q. intricata & Q. subspathulata \\
Q. emoryi & Q. viminea & Q. laeta & Q. tuberculata \\
Q. fulva & & Q. magnoliifolia & \\
\hline
\end{tabular}


términos generales son más accesibles de medir y menos costosas que los ensayos físico y mecánicos, los cuales en su mayoría requieren de mayor material biológico y de equipo caro. Resultaría muy útil poder inferir las propiedades tecnológicas de la madera a partir de la anatomía de todas las especies, o por lo menos de varias o de ciertos caracteres anatómicos con algunas propiedades.

La necesidad de conocer a los encinos mexicanos abarca disciplinas como la taxonomía, ecología, en particular sobre sus poblaciones, tecnología, fisiología, mercadotecnia, sustentabilidad, restauración, etc. El estudio y el conocimiento de las características anatómicas y tecnológicas de la madera, son herramientas valiosas que ayudan a su mejor aprovechamiento y permiten dar a cada especie o grupo de especies, los usos más acordes con sus propiedades (Wangaard, 1981; De la Paz Pérez et al, 1997). Sin embargo, los estudios del género Quercus sobre este tema son escasos en México. Como trabajo pionero está el de Negrete (1970) que hizo la relación de la longitud de las fibras con algunas características físicas de cuatro encinos de Michoacán. Posteriormente se ha estudiado con frecuencia la relación entre la estructura y las propiedades mecánicas de la madera. Entre las variables más importantes de las que se ha determinado su influencia están las fibras y los radios. De la Paz Pérez y Dávalos (1995), analizaron la relación de las características anatómicas con las propiedades físicas y mecánicas de dos especies de Quercus recolectadas en Puebla; Guerrero et al. (1995), hicieron la relación de la estructura anatómica de Quercus sartorii con dos propiedades físicas y dos propiedades mecánicas; de la Paz Pérez (2000) relacionó doce variables anatómicas con las propiedades físicas y mecánicas de 24 especies de Quercus recolectadas en varios estados de la República.

\section{OBJETIVO}

Determinar la relación entre el parénquima radial (radios uniseriados y multiseriados) y la contracción volumétrica (propiedad física), la flexión (MOR y MOE), la compresión paralela (EMAX) y perpendicular (ELP) y el cortante paralelo (EMAX) (propiedades mecánicas), de la madera de ocho especies de Quercus de Durango: $Q$. candicans, Q. coccolobifolia, Q. crassifolia, $Q$. durifolia, Q. sideroxyla (encinos rojos), $Q$. convallata, Q. laeta y $Q$. potosina (encinos blancos), recolectadas en tres municipios.

\section{MATERIAL Y MÉTODOS}

Las especies estudiadas, se recolectaron en los municipios de San Dimas, Pueblo Nuevo y Durango, del estado de Durango (Fig. 1). Se seleccionó un árbol por especie, de fuste recto, de cuando menos 40 $\mathrm{cm}$ de diámetro. Los ejemplares de herbario se registraron en el Herbario del Instituto Nacional de Investigaciones Forestales "Luciano Vela" y las muestras de xiloteca en la Xiloteca "Juana Huerta", del mismo Instituto (Tabla 2). De cada árbol se cortaron cuatro trozas, de donde se tomó el material para el estudio anatómico, físico y mecánico. De la primera troza se obtuvo el material para el estudio de los radios, de las tres restantes se hicieron tablones en sentido tangencial y radial de los que se obtuvieron las probetas para la contracción volumétrica, de las mismas trozas se sacaron prismas de los que se obtuvieron las diferentes probetas para los ensayos mecánicos (Fig. 2). Las pruebas físicas y mecánicas, se hicieron en condición 


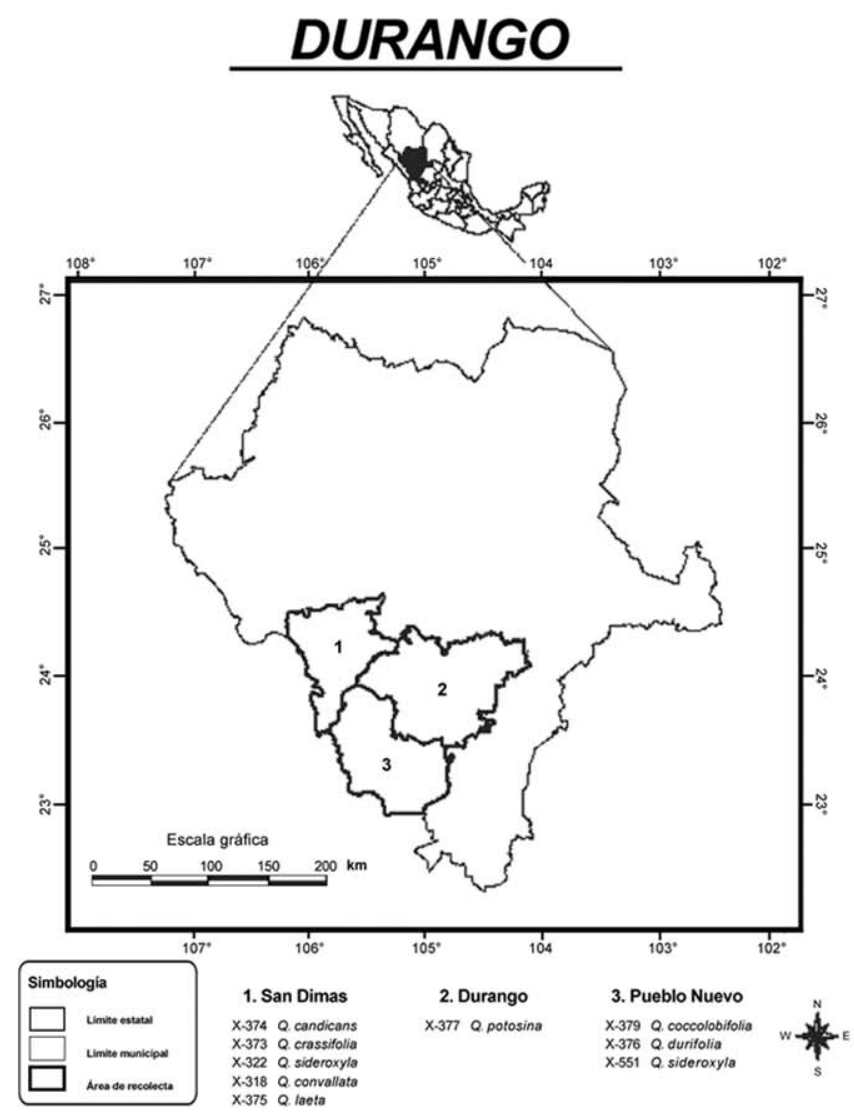

Fig. 1 Sitios de recolección de las especies estudiadas.

verde, a un contenido de humedad mayor de $30 \%$. Se realizaron ensayes de cortante paralelo a la fibra, compresión paralela y perpendicular a la fibra y flexión estática. Para la contracción volumétrica y los ensayos mecánicos se elaboraron probetas de diferentes tamaños, de acuerdo con la norma ASTM-D-143 (ASTM 1993). Se utilizó el método primario de ensaye de probetas pequeñas libres de defectos. Se probaron seis especimenes por tipo de ensaye por especie; las dimensiones de la sección transversal de cada probeta fueron de $5 \mathrm{~cm} x$ $5 \mathrm{~cm}$ y las longitudes fueron variables, de acuerdo con el tipo de ensaye. Parte de la determinación de las características físicas y mecánicas se llevó a cabo en el Laboratorio de la Unidad de Recursos Forestales del Instituto de Ecología A. C. de Xalapa, Ver. Algunos datos fueron proporcionados por el Ing. Francisco Robles Gálvez, anteriormente investigador del INIF.

La altura y número por $5 \mathrm{~mm}$ de los radios multiseriados, se midieron en tablillas tangenciales de $15 \mathrm{~cm} \times 7 \mathrm{~cm} \times 1 \mathrm{~cm}$, la anchura y número de series de los radios multiseriados y el número de los radios uniseriados por $\mathrm{mm}$, se midieron en preparaciones fijas de cortes tangenciales. El 

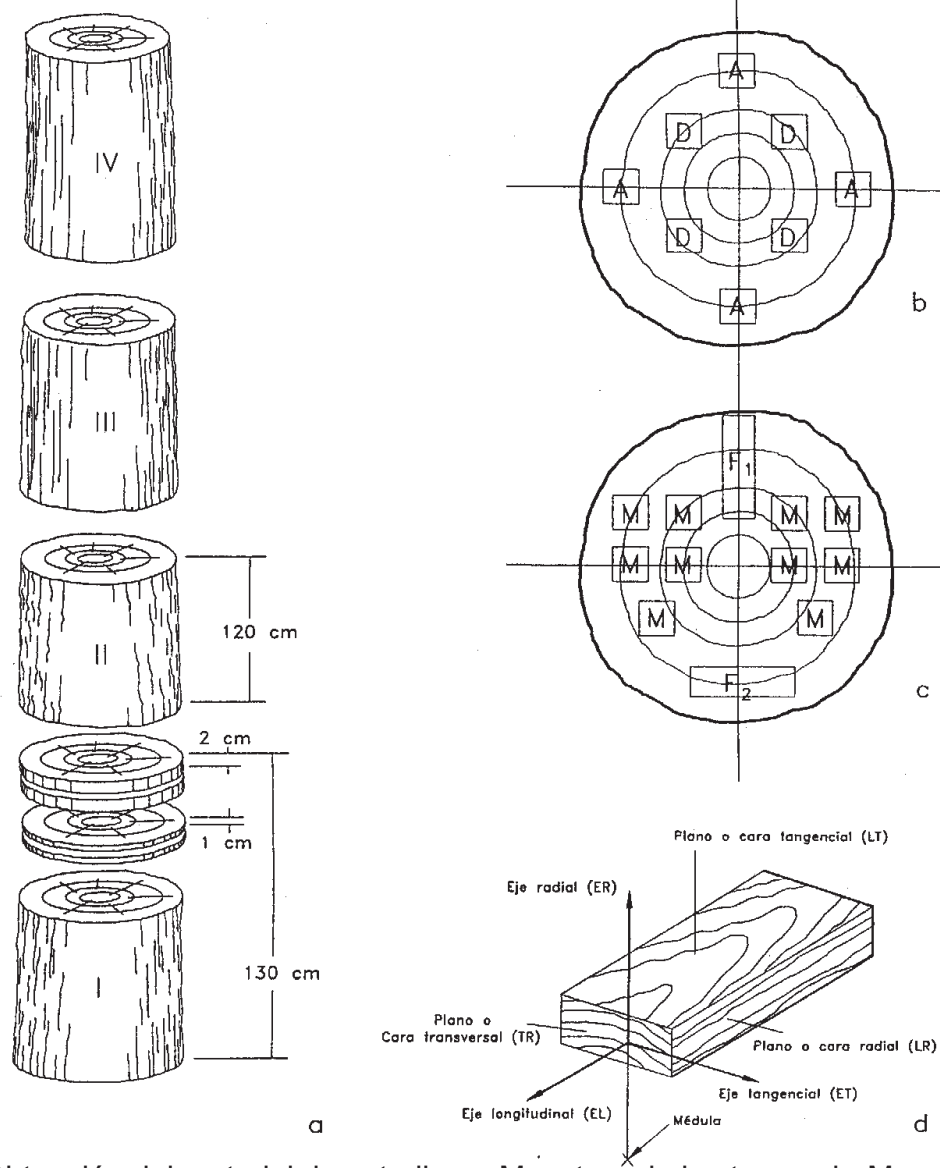

Fig. 2 Obtención del material de estudio. a. Muestreo de las trozas. b. Muestreo de los cubos $\left(A=\right.$ altura. $D=$ duramen). $c$. Muestreo de los tablones $\left(F_{1}=\right.$ radial. $F_{2}=$ tangencial $)$ y prismas (M). d. Ejes y planos de la madera.

estudio anatómico se realizó en el Laboratorio de Anatomía y Tecnología de la Universidad Autónoma Metropolitana unidad Iztapalapa. A todos los caracteres se les hizo un análisis estadístico univariado y se determinaron con base en la media. Para los radios se utilizaron las tablas de Chattaway (1932) y IAWA Committee $(1939,1989)$, para la contracción volumétrica a Echenique et al. (1975) y para las propiedades mecánicas a Dávalos y Bárcenas (1998) (Anexo 1).

\section{RESULTADOS}

Los radios de las especies estudiadas muestran diferencias anatómicas considerables entre los ejemplares de los distintos subgéneros en la anchura, el número de series y número por 5 milímetros de los radios multiseriados, presentando los valores más altos los encinos blancos. Por otro lado, el número de radios uniseriados por milímetro y la altura de los radios multiseriados, no presentan diversidad notable entre las especies de ambos 
subgéneros, excepto $Q$. potosina, que tiene radios excesivamente altos (Tabla 3, Láminas 1 y 2). En cuanto a las características físicas (contracción volumétrica) y las mecánicas (flexión, compresión y cortante) ensayadas, los valores más altos también se presentan en los encinos blancos (Tabla 4).

A través de un análisis multivariado, en donde los valores de los radios fueron las variables dependientes y las propiedades físicas y mecánicas las variables independientes, se obtuvo la información de la influencia de los radios con las propiedades tecnológicas ensayadas. Debido a que se trata de un número relativamente reducido de datos (puntos de las gráficas), no se hicieron análisis estadísticos más sofisticados como ANOVA, Mann-Whitney o correlación canónica, pues lo que se pretende es establecer la tendencia existente, para lo cual los análisis de regresión múltiple paso a paso son razonablemente útiles. Los resultados se presentan en tablas y gráficas y se ilustran con fotografías.

Los resultados del análisis de regresión, que se presentan en la Tabla 5 y de manera gráfica en las Figs. 3 a 6 , mostraron que las características anatómicas del parénquima radial con más influencia en las propiedades tecnológicas son la anchura y la altura de los radios multiseriados, ya que intervienen en la mayoría de ellas: en la contracción, en la flexión MOR y MOE, en la compresión paralela y el cortante. Además, el número de series, también contribuyen en la contracción y el número por cinco milímetros, en el cortante paralelo. Por otra parte, el efecto del número de radios uniseriados por milímetro es definitivo en la compresión paralela (Tablas 6 y 7 ).

Tabla 2. Registro de las especies estudiadas

\begin{tabular}{|c|c|c|c|c|c|c|}
\hline \multirow[t]{2}{*}{ ESPECIES } & \multicolumn{2}{|c|}{ MUNICIPIO ALTITUDms } & $\mathrm{nm}$ & PROVINCIA & HERBARIO & \multirow[t]{2}{*}{ XILOTECA } \\
\hline & \multicolumn{5}{|c|}{ ENCINOS ROJOS } & \\
\hline Q. candicans & San Dimas & 2400 & & ierra Madre Occidental & 22684 & $X-374$ \\
\hline Q. coccolobifolia & Pueblo Nuevo & 2380 & & ierra Madre Occidental & 22682 & $X-379$ \\
\hline Q. crassifolia & San Dimas & 2400 & & ierra Madre Occidental & 20759 & $X-373$ \\
\hline Q. durifolia & Pueblo Nuevo & 2350 & & ierra Madre Occidental & 22685 & $X-376$ \\
\hline Q. sideroxyla & San Dimas & 2600 & & ierra Madre Occidental & 18820 & $X-322$ \\
\hline \multirow[t]{2}{*}{ Q. sideroxyla } & Pueblo Nuevo & 2786 & & ierra Madre Occidental & 33086 & $X-551$ \\
\hline & \multicolumn{6}{|c|}{ ENCINOS BLANCOS } \\
\hline Q. convallata & San Dimas & 2480 & & ierra Madre Occidental & 18814 & $x-318$ \\
\hline Q. laeta & San Dimas & 2400 & & ierra Madre Occidental & 20760 & $X-375$ \\
\hline Q. potosina & Durango & 2380 & & ierra Madre Occidental & 20761 & $\mathrm{X}-377$ \\
\hline
\end{tabular}


Tabla 3. Valores anatómicos

\begin{tabular}{|c|c|c|c|c|c|}
\hline \multirow{3}{*}{ ESPECIES } & \multicolumn{5}{|c|}{ RADIOS } \\
\hline & \multirow{2}{*}{$\begin{array}{c}\text { UNISERIADOS } \\
\text { número x mm }\end{array}$} & \multicolumn{4}{|c|}{ MULTISERIADOS } \\
\hline & & $\begin{array}{l}\text { número } \\
\times 5 \mathrm{~mm}\end{array}$ & $\begin{array}{l}\text { altura } \\
(\mathrm{cm})\end{array}$ & $\begin{array}{c}\text { anchura } \\
(\mu)\end{array}$ & $\begin{array}{l}\text { número } \\
\text { de series }\end{array}$ \\
\hline \multicolumn{6}{|c|}{ ENCINOS ROJOS } \\
\hline $\begin{array}{l}\text { Q. candicans } \\
\text { San Dimas }\end{array}$ & $\begin{array}{c}\text { Numerosos } \\
9\end{array}$ & $\begin{array}{c}\text { pocos } \\
2\end{array}$ & $\begin{array}{l}\text { altos } \\
2,0\end{array}$ & $\begin{array}{c}\text { ext anchos } \\
482\end{array}$ & 25 \\
\hline $\begin{array}{l}\text { Q. coccolobifolia } \\
\text { Pueblo Nuevo }\end{array}$ & $\begin{array}{c}\text { Numerosos } \\
9\end{array}$ & $\begin{array}{c}\text { pocos } \\
2\end{array}$ & $\begin{array}{c}\text { altos } \\
1,8\end{array}$ & $\begin{array}{c}\text { ext. anchos } \\
470\end{array}$ & 24 \\
\hline $\begin{array}{l}\text { Q. crassifolia } \\
\text { San Dimas }\end{array}$ & $\begin{array}{c}\text { Numerosos } \\
8\end{array}$ & $\begin{array}{c}\text { pocos } \\
2\end{array}$ & $\begin{array}{c}\text { altos } \\
1,5\end{array}$ & $\begin{array}{c}\text { muy anchos } \\
387\end{array}$ & 20 \\
\hline $\begin{array}{l}\text { Q. durifolia } \\
\text { Pueblo Nuevo }\end{array}$ & $\begin{array}{c}\text { Numerosos } \\
10\end{array}$ & $\begin{array}{c}\text { pocos } \\
2\end{array}$ & $\begin{array}{c}\text { muy altos } \\
2,5\end{array}$ & $\begin{array}{c}\text { muy anchos } \\
362\end{array}$ & 19 \\
\hline $\begin{array}{l}\text { Q. sideroxyla } \\
\text { San Dimas }\end{array}$ & $\begin{array}{c}\text { muy numerosos } \\
11\end{array}$ & $\begin{array}{c}\text { pocos } \\
2\end{array}$ & $\begin{array}{c}\text { muy altos } \\
2,5\end{array}$ & $\begin{array}{c}\text { muy anchos } \\
386\end{array}$ & 20 \\
\hline $\begin{array}{l}\text { Q. sideroxyla } \\
\text { Pueblo Nuevo }\end{array}$ & $\begin{array}{c}\text { Numerosos } \\
10\end{array}$ & $\begin{array}{c}\text { pocos } \\
2\end{array}$ & $\begin{array}{c}\text { altos } \\
2,0\end{array}$ & $\begin{array}{l}\text { ext. anchos } \\
455\end{array}$ & 24 \\
\hline \multicolumn{6}{|c|}{ ENCINOS BLANCOS } \\
\hline $\begin{array}{l}\text { Q. convallata } \\
\text { San Dimas }\end{array}$ & $\begin{array}{c}\text { muy numerosos } \\
12\end{array}$ & $\begin{array}{c}\text { pocos } \\
1\end{array}$ & $\begin{array}{c}\text { altos } \\
2,0\end{array}$ & $\begin{array}{l}\text { ext. anchos } \\
952\end{array}$ & 50 \\
\hline $\begin{array}{l}\text { Q. laeta } \\
\text { San Dimas }\end{array}$ & $\begin{array}{c}\text { muy numerosos } \\
11\end{array}$ & $\begin{array}{c}\text { pocos } \\
1\end{array}$ & $\begin{array}{c}\text { muy altos } \\
2,5\end{array}$ & $\begin{array}{l}\text { ext. anchos } \\
600\end{array}$ & 31 \\
\hline $\begin{array}{l}\text { Q. potosina } \\
\text { Durango }\end{array}$ & $\begin{array}{c}\text { muy numerosos } \\
11\end{array}$ & $\begin{array}{c}\text { pocos } \\
1\end{array}$ & $\begin{array}{c}\text { Ext. altos } \\
5,0\end{array}$ & $\begin{array}{l}\text { ext. anchos } \\
793\end{array}$ & 24 \\
\hline
\end{tabular}


Tabla 4. Valores físicos y mecánicos

\begin{tabular}{|c|c|c|c|c|c|c|}
\hline \multirow{3}{*}{ ESPECIES } & \multirow{3}{*}{$\begin{array}{c}\text { CONTRACCIÓN } \\
\text { VOLUMÉTRICA } \\
(\%)\end{array}$} & \multirow{2}{*}{\multicolumn{2}{|c|}{$\begin{array}{l}\text { FLEXIÓN } \\
\text { ESTÁTICA }\end{array}$}} & \multicolumn{2}{|c|}{ COMPRESIÓN } & \multirow{3}{*}{$\begin{array}{c}\text { CORTANTE } \\
\begin{array}{l}\text { EMAX } \\
\text { (MPa) }\end{array}\end{array}$} \\
\hline & & & & \multirow{2}{*}{$\begin{array}{l}\text { PARALELA } \\
\text { EMAX } \\
\text { (MPa) }\end{array}$} & \multirow{2}{*}{$\begin{array}{c}\text { PERPEN } \\
\text { DICULAR } \\
\text { ELP } \\
(\mathrm{MPa})\end{array}$} & \\
\hline & & $\begin{array}{l}\text { MOR } \\
(\mathrm{MPa})\end{array}$ & $\begin{array}{l}\mathrm{MOE} \\
(\mathrm{MPa})\end{array}$ & & & \\
\hline \multicolumn{7}{|c|}{ ERYTHROBALANUS (ENCINOS ROJOS) } \\
\hline $\begin{array}{l}\text { Q.candicans } \\
\text { San Dimas }\end{array}$ & $\begin{array}{l}\text { alta } \\
17,07\end{array}$ & $\begin{array}{l}\text { medio } \\
63,4\end{array}$ & $\begin{array}{c}\text { medio } \\
9679\end{array}$ & $\begin{array}{c}\text { Medio } \\
27,7\end{array}$ & $\begin{array}{l}\text { alto } \\
6,4\end{array}$ & $\begin{array}{c}\text { Alto } \\
9,2\end{array}$ \\
\hline $\begin{array}{l}\text { Q. } \\
\text { coccolobifolia } \\
\text { Pueblo } \\
\text { Nuevo }\end{array}$ & $\begin{array}{c}\text { muy alta } \\
20,09\end{array}$ & $\begin{array}{l}\text { alto } \\
65,1\end{array}$ & $\begin{array}{l}\text { alto } \\
9895\end{array}$ & $\begin{array}{l}\text { Medio } \\
22,4\end{array}$ & $\begin{array}{l}\text { alto } \\
6,7\end{array}$ & $\begin{array}{c}\text { Alto } \\
9,4\end{array}$ \\
\hline $\begin{array}{l}\text { Q. crassifolia } \\
\text { San Dimas }\end{array}$ & $\begin{array}{c}\text { muy alta } \\
20,21\end{array}$ & $\begin{array}{l}\text { alto } \\
69,4\end{array}$ & $\begin{array}{c}\text { alto } \\
10415\end{array}$ & $\begin{array}{l}\text { Medio } \\
29,7\end{array}$ & $\begin{array}{l}\text { alto } \\
7,6\end{array}$ & $\begin{array}{c}\text { Alto } \\
9,7\end{array}$ \\
\hline $\begin{array}{l}\text { Q. durifolia } \\
\text { Pueblo } \\
\text { Nuevo }\end{array}$ & $\begin{array}{c}\text { muy alta } \\
19,23\end{array}$ & $\begin{array}{l}\text { Alto } \\
85,1\end{array}$ & $\begin{array}{l}\text { muy } \\
\text { alto } \\
12484\end{array}$ & $\begin{array}{l}\text { Alto } \\
35,6\end{array}$ & $\begin{array}{c}\text { muy alto } \\
11,4\end{array}$ & $\begin{array}{c}\text { muy alto } \\
11,4\end{array}$ \\
\hline $\begin{array}{l}\text { Q. sideroxyla } \\
\text { San Dimas }\end{array}$ & $\begin{array}{c}\text { alta } \\
18,48\end{array}$ & $\begin{array}{l}\text { Alto } \\
65,2\end{array}$ & $\begin{array}{l}\text { alto } \\
9905\end{array}$ & $\begin{array}{l}\text { Medio } \\
28,7\end{array}$ & $\begin{array}{c}\text { muy alto } \\
10,7\end{array}$ & $\begin{array}{c}\text { Alto } \\
9,2\end{array}$ \\
\hline $\begin{array}{l}\text { Q. sideroxyla } \\
\text { Pueblo } \\
\text { Nuevo }\end{array}$ & $\begin{array}{c}\text { alta } \\
16,83\end{array}$ & $\begin{array}{l}\text { Alto } \\
66,8\end{array}$ & $\begin{array}{l}\text { alto } \\
10091\end{array}$ & $\begin{array}{l}\text { Medio } \\
28,7\end{array}$ & $\begin{array}{l}\text { alto } \\
7,0\end{array}$ & $\begin{array}{c}\text { Alto } \\
9,5\end{array}$ \\
\hline \multicolumn{7}{|c|}{ LEUCOBALANUS (ENCINOS BLANCOS) } \\
\hline $\begin{array}{l}\text { Q. convallata } \\
\text { San Dimas }\end{array}$ & $\begin{array}{c}\text { muy alta } \\
19,51\end{array}$ & $\begin{array}{l}\text { Alto } \\
79,5\end{array}$ & $\begin{array}{l}\text { alto } \\
11601\end{array}$ & $\begin{array}{l}\text { Alto } \\
31,2\end{array}$ & $\begin{array}{c}\text { muy alto } \\
16,0\end{array}$ & $\begin{array}{c}\text { muy alto } \\
11,4\end{array}$ \\
\hline $\begin{array}{l}\text { Q. laeta } \\
\text { San Dimas }\end{array}$ & $\begin{array}{c}\text { muy alta } \\
21,44\end{array}$ & $\begin{array}{l}\text { Alto } \\
84,2\end{array}$ & $\begin{array}{l}\text { alto } \\
12150\end{array}$ & $\begin{array}{l}\text { Alto } \\
34,7\end{array}$ & $\begin{array}{l}\text { Alto } \\
10,7\end{array}$ & $\begin{array}{c}\text { muy alto } \\
11,9\end{array}$ \\
\hline $\begin{array}{l}\text { Q. potosina } \\
\text { Durango }\end{array}$ & $\begin{array}{c}\text { muy alta } \\
22,51\end{array}$ & $\begin{array}{l}\text { Alto } \\
86,5\end{array}$ & $\begin{array}{c}\text { muy } \\
\text { alto } \\
12494\end{array}$ & $\begin{array}{l}\text { Alto } \\
35,7\end{array}$ & $\begin{array}{c}\text { muy alto } \\
11,4\end{array}$ & $\begin{array}{c}\text { muy alto } \\
12,3\end{array}$ \\
\hline
\end{tabular}




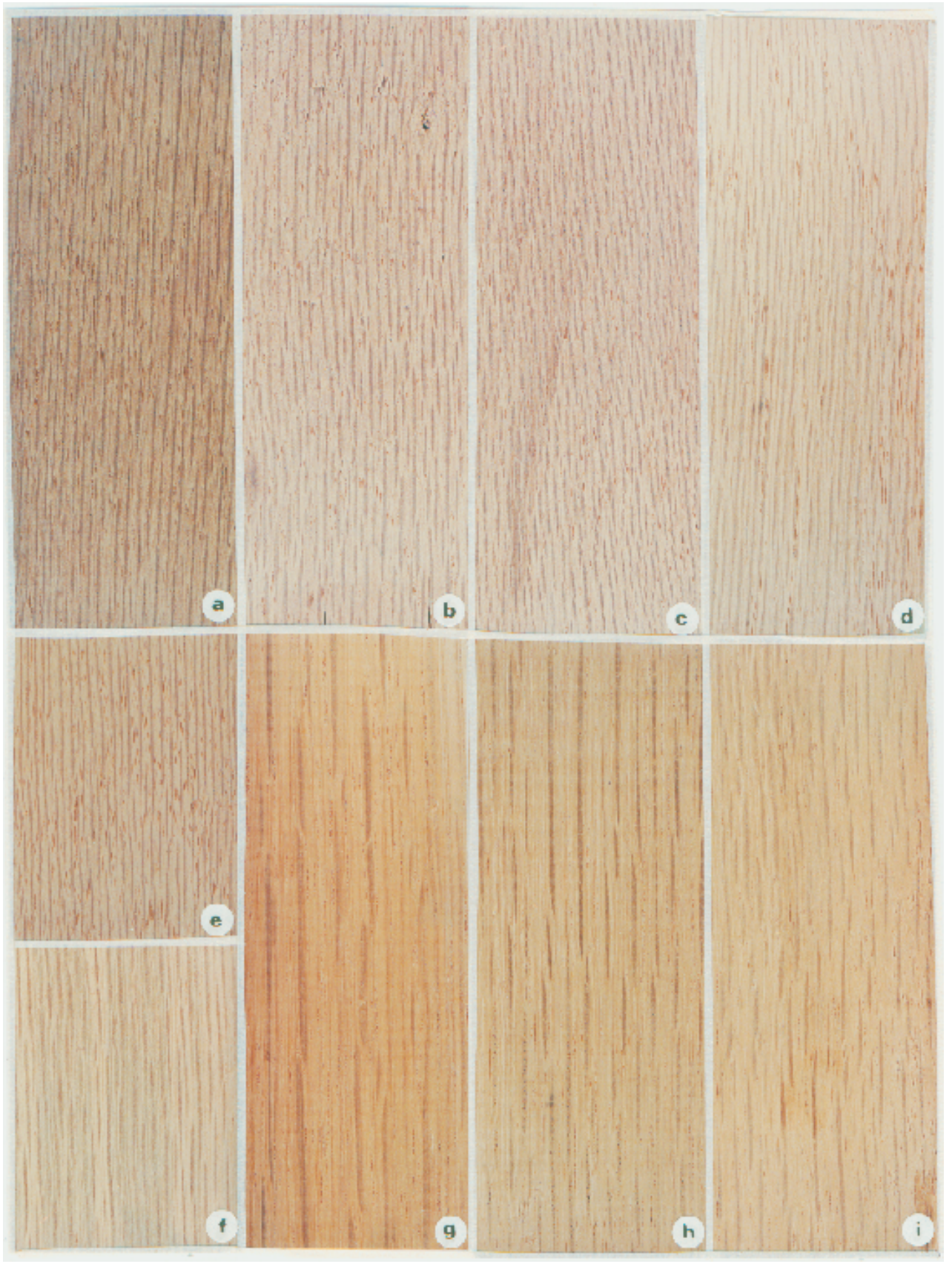

Lám. 1. Tablillas tangenciales. a. Quercus candicans. b. Q. Cocolobifolia. c. Q. Crassifolia. d Q. Durifolia. e-f. Q. Sideroxyla (encinos rojos). g. Q. Convallata. h. Q laeta. i. Q. Potosina (encinos blancos). 


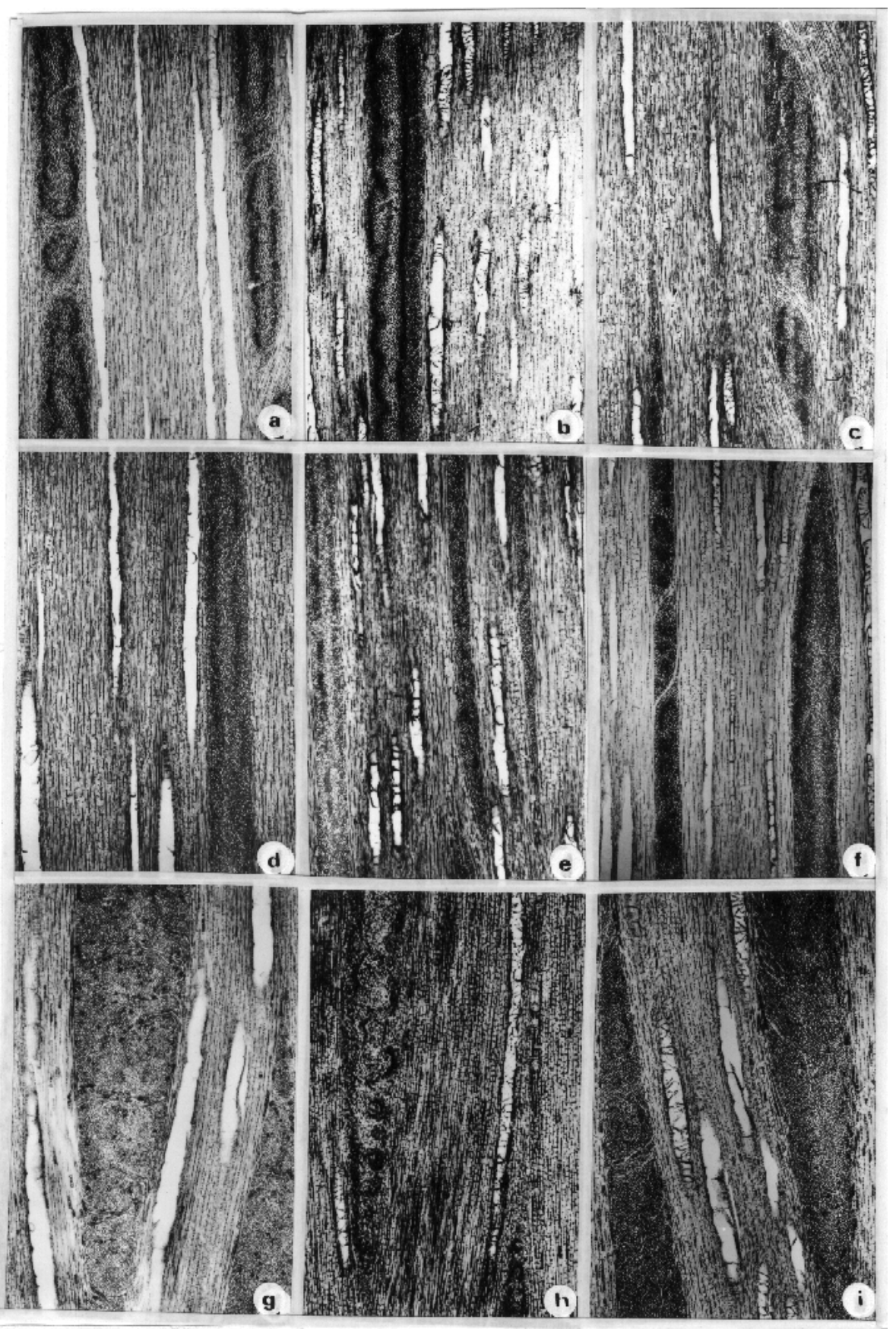

Lám. 2. Cortes tangenciales. a. Quercus candicans. b. Q. Cocolobifolia. c. Q. Crassifolia. d Q. Durifolia. e-f. Q. Sideroxyla (encinos rojos). g. Q. Convallata. h. Q laeta. i. Q. Potosina (encinos blancos) (18x). 


\section{DISCUSIÓN}

Los radios multiseriados son los elementos más distintivos de la madera de encino y junto con los radios uniseriados tienen gran influencia en las propiedades tecnológicas de la misma (Mckintosh, 1954; Linsay y Chalk, 1954; de la Paz Pérez et al., 1997). Las características anatómicas de los radios multiseriados de los encinos, favorecen las contracciones, que es uno de los mayores problemas que se presenta en su industrialización, principalmente en el secado (Ávila, 1985; Montes, 1985), por lo que en sus procesos de transformación, es indispensable seleccionar a las especies que presenten valores bajos de estos elementos, principalmente en su altura, su anchura y número de series o al menos, separar la madera de acuerdo con las características de los radios. Las diferencias entre subgéneros son más notables que entre las especies del mismo subgénero (de la Paz Pérez y Aguilar, 1978).

Maeglin y Quirk (1984), estudiaron el comportamiento de la madera de los encinos rojos y blancos en relación con el porcentaje y dimensiones de los elementos constitutivos, entre los que destacaron a los radios multiseriados. Los resultados obtenidos por de la Paz Pérez (2000) (Tabla 5), señalan que las variables anatómicas que mas influyen en el comportamiento físico y mecánico de la madera de estos encinos, son las dimensiones de las fibras y las características de los radios multiseriados. Los valores incluidos en esta tabla corresponden a los coeficientes de las variables incluidas (dependientes e independientes) en la regresión, que aparecen en el primer renglón de cada grupo de resultados por especie. En

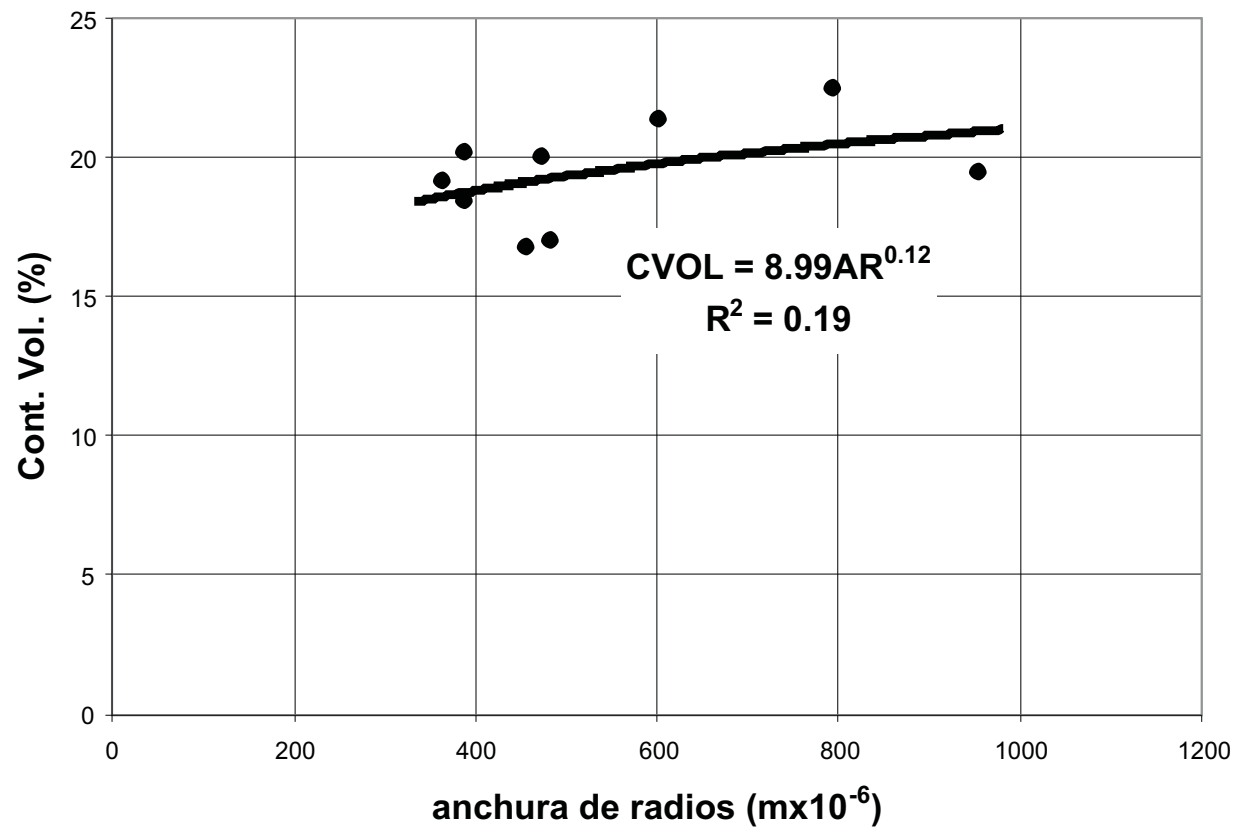

Fig. 3 Curva de regresión de la anchura de los radios multiseriados y la contracción volumétrica 


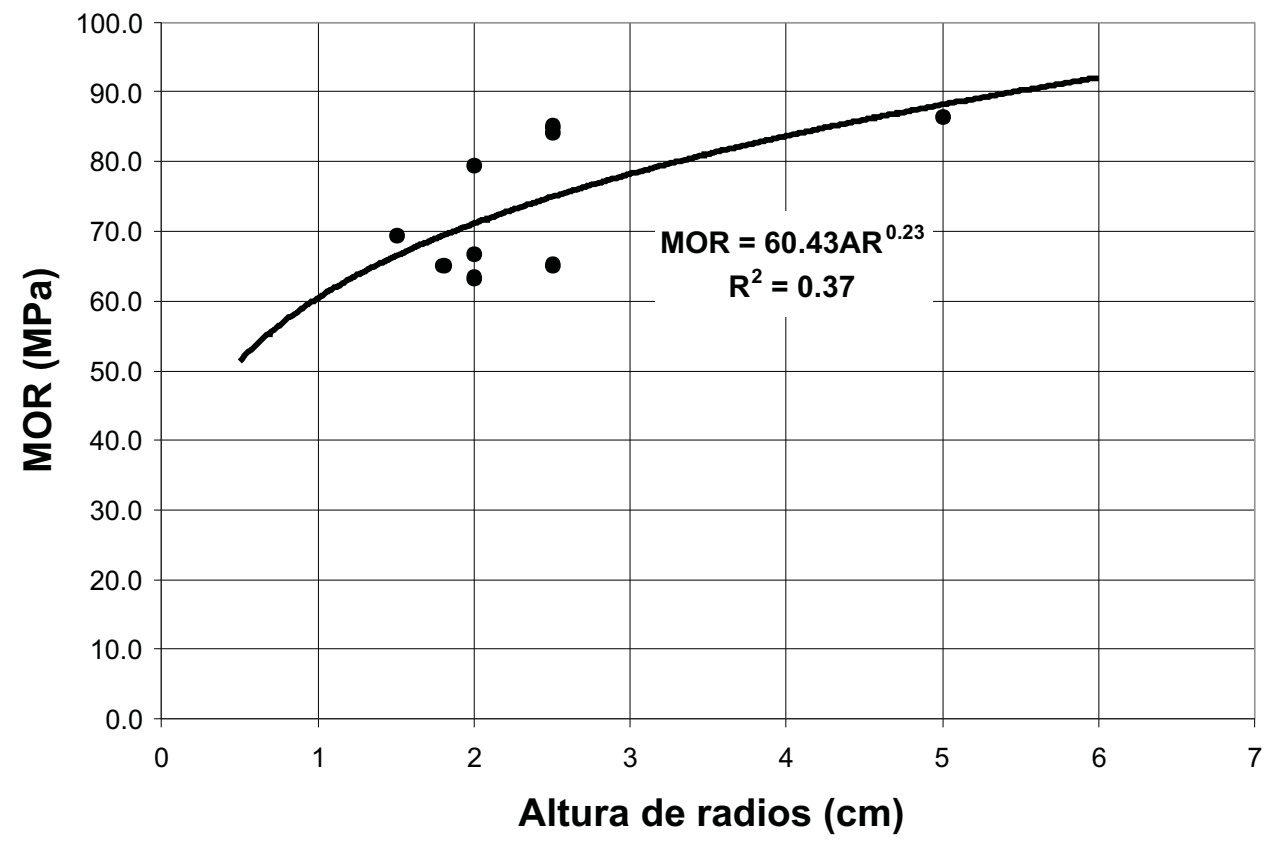

Fig. 4 Curva de regresión de la altura de los radios multiseriados y la flexión MOR

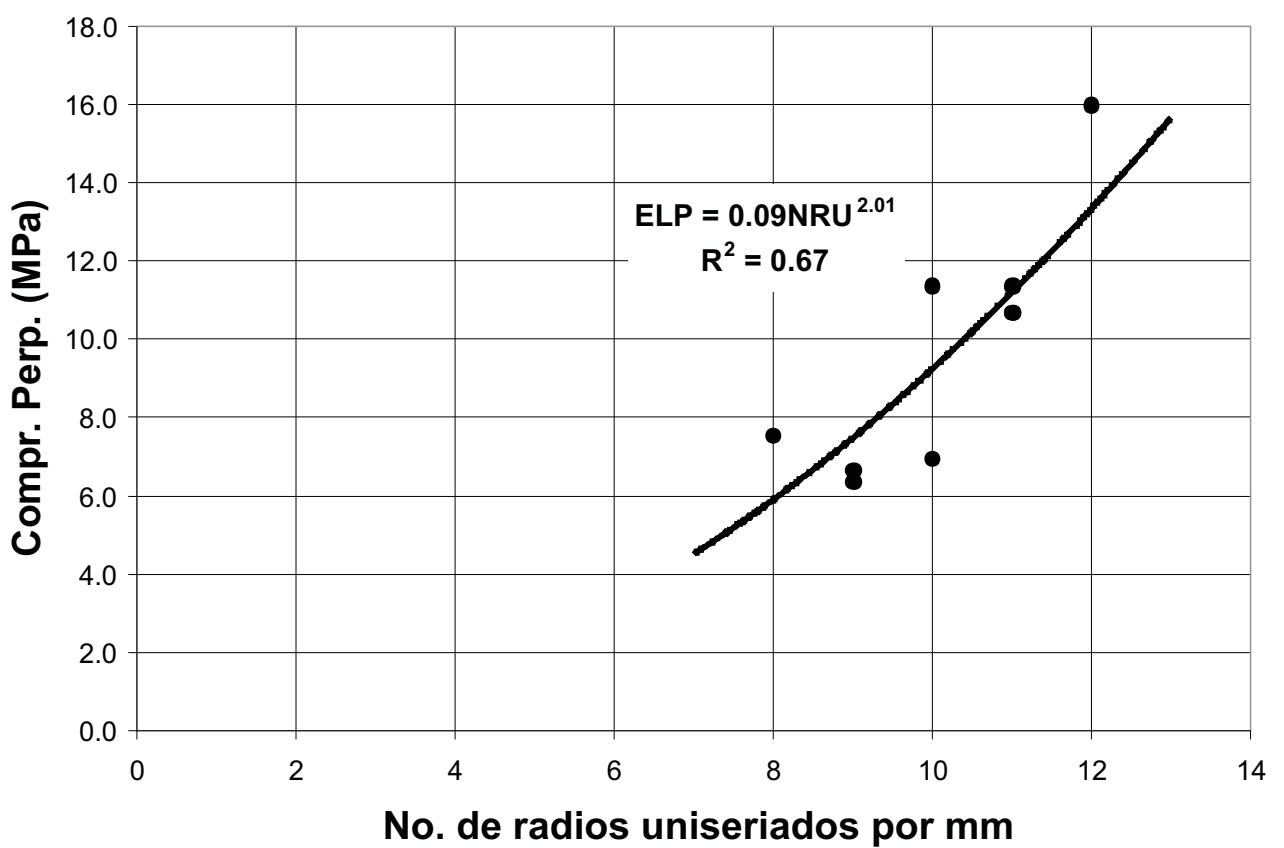

Fig. 5 Curva de regresión de los radios uniseriados y la compresión perpendicular ELP 
Tabla 5. Análisis de regresión con varios elementos anatómicos (de la Paz Pérez 2000)

\begin{tabular}{|c|c|c|c|c|c|}
\hline \multicolumn{6}{|c|}{ Contracción volumétrica (\%) } \\
\hline & $\begin{array}{l}\text { grosor } \\
\text { de fibras }\end{array}$ & $\begin{array}{l}\text { diámetro } \\
\text { de fibras }\end{array}$ & $\begin{array}{c}\text { series } \\
\text { de radios }\end{array}$ & $\begin{array}{l}\text { Anchura } \\
\text { de radios }\end{array}$ & b \\
\hline Coeficientes & $-0,641$ & $-0,244$ & $-4,891$ & 0,262 & 18,26 \\
\hline Error estándar & 0,664 & 0,734 & 2,037 & 0,108 & 6,63 \\
\hline Coef. Det. $\left(R^{2}\right)$ & 0.68 & 1.50 & & & \\
\hline \multicolumn{6}{|c|}{ Flexión MOR (MPa) } \\
\hline & $\begin{array}{l}\text { grosor } \\
\text { de fibras }\end{array}$ & $\begin{array}{l}\text { Anchura } \\
\text { de radios }\end{array}$ & $\begin{array}{c}\text { altura de } \\
\text { radios }(\mathrm{cm})\end{array}$ & longitud fibras & $\mathrm{b}$ \\
\hline Coeficientes & $-3,394$ & 0,011 & 4,555 & 0,028 & 40.27 \\
\hline Error estándar & 3,125 & $\begin{array}{l}0,015 \\
7769\end{array}$ & 3,340 & 0,017 & 30,39 \\
\hline \multicolumn{6}{|c|}{ flexión MOE (Mpa) } \\
\hline & $\begin{array}{l}\text { grosor } \\
\text { de fibras }\end{array}$ & $\begin{array}{l}\text { Anchura } \\
\text { de radios }\end{array}$ & $\begin{array}{l}\text { longitud de } \\
\text { radios }(\mathrm{cm})\end{array}$ & longitud fibras & b \\
\hline Coeficientes & $-444,3$ & 1,14 & & & \\
\hline Error estándar & 389,9 & 1,89 & 562,3 & 3,51 & 7081,6 \\
\hline Coef. Det. $\left(R^{2}\right)$ & 0,671 & 969,4 & 416,7 & 2,13 & 3792,6 \\
\hline \multicolumn{6}{|c|}{ Compresión paralela EMAX (Mpa) } \\
\hline & $\begin{array}{l}\text { diámetro } \\
\text { de fibras }\end{array}$ & $\begin{array}{l}\text { Grosor } \\
\text { de fibras }\end{array}$ & $\begin{array}{l}\text { longitud de } \\
\text { radios }(\mathrm{cm})\end{array}$ & $\begin{array}{l}\text { longitud } \\
\text { de vasos }\end{array}$ & b \\
\hline Coeficientes & $-0,766$ & $-0,734$ & & & \\
\hline Error estándar & 0,699 & 1,016 & 1,648 & 0,026 & $\begin{array}{l}25,142 \\
10703\end{array}$ \\
\hline Coef. Det. $\left(R^{2}\right)$ & 0,700 & 2,564 & & & \\
\hline \multicolumn{6}{|c|}{ Compresión perpendicular ELP (Mpa) } \\
\hline & $\begin{array}{l}\text { diámetro } \\
\text { de fibras }\end{array}$ & No. Poros & $\begin{array}{l}\text { Grosor } \\
\text { de fibras }\end{array}$ & $\begin{array}{c}\mathrm{No} / \mathrm{mm} \\
\mathrm{RU}\end{array}$ & $b$ \\
\hline Coeficientes & $-0,340$ & $-0,515$ & & & \\
\hline Error estándar & 0,626 & 0,484 & 0,205 & $1, / 42$ & $-3,407$ \\
\hline Coef. Det. $\left(R^{2}\right)$ & 0,777 & 2,106 & & & 13,635 \\
\hline \multicolumn{6}{|c|}{ Cortante EMAX (Mpa) } \\
\hline & $\begin{array}{c}\text { Grosor } \\
\text { de fibras }\end{array}$ & $\begin{array}{l}\text { diámetro } \\
\text { de fibras }\end{array}$ & $\begin{array}{l}\text { No/5mm } \\
\text { RM }\end{array}$ & $\begin{array}{l}\text { longitud de } \\
\text { radios }(\mathrm{cm})\end{array}$ & b \\
\hline Coeficientes & $-0,240$ & $-0,031$ & & & \\
\hline Error estándar & 0,357 & 0,314 & 1024 & 0,387 & $\begin{array}{l}14,942 \\
3,456\end{array}$ \\
\hline Coef. Det. $\left(R^{2}\right)$ & 0,785 & 0,821 & & & \\
\hline
\end{tabular}


Tabla 6. Análisis de regresión únicamente con radios

Contracción Volumétrica (\%)

\begin{tabular}{cccrr}
\hline & No. Radios & Altura & Anchura & \multicolumn{1}{c}{ B } \\
\hline Coeficientes & $-4,073$ & 0,468 & $-0,006$ & 28,32 \\
Error estándar & 2,395 & 0,612 & 0,005 & 7,30 \\
Coef. Determ. y Error Estim. & $\mathbf{0 , 6 0 8}$ & 1,476 & &
\end{tabular}

\begin{tabular}{cccrr}
\hline \multicolumn{5}{c}{ flexión MOR (Mpa) } \\
\hline & No./5mm & Altura & Anchura & \multicolumn{1}{c}{ B } \\
\hline Coeficientes & $-21,10$ & 2,31 & $-0,026$ & 117,79 \\
Error estándar & 11,59 & 2,96 & 0,026 & 35,35 \\
Coef. Determ. y Error Estim. & $\mathbf{0 , 6 6 3}$ & $\mathbf{7 , 1 5}$ & & \\
\end{tabular}

\begin{tabular}{ccccc}
\hline \multicolumn{5}{c}{ flexión MOE (Mpa) } \\
\hline & No./5mm & Altura & Anchura & B \\
\hline Coeficientes & $-2481,4$ & 309,7 & $-3,23$ & 16106,8 \\
Error estándar & 1506,3 & 384,9 & 3,42 & 4592,8 \\
Coef. Determ. y Error Estim. & $\mathbf{0 , 6 2 3}$ & $\mathbf{9 2 8 , 3}$ & &
\end{tabular}

\begin{tabular}{ccccc}
\hline \multicolumn{5}{c}{ compresión paralela EMAX (Mpa) } \\
\hline & No./5mm & Altura & Anchura & B \\
\hline Coeficientes & $-9,48$ & 1,43 & $-0,017$ & 51,95 \\
Error estándar & 5,76 & 1,47 & 0,013 & 17,57 \\
Coef. Determ. y Error Estim. & $\mathbf{0 , 5 8 6}$ & $\mathbf{3 , 5 5}$ & & \\
& & & & \\
\hline
\end{tabular}

\begin{tabular}{|c|c|c|c|c|}
\hline \multicolumn{5}{|c|}{ compresión perpendicular ELP (Mpa } \\
\hline & No./5mm & Altura & Anchura & $\mathrm{B}$ \\
\hline Coeficientes & $-2,61$ & $-0,16$ & 0,006 & 11,47 \\
\hline Error estándar & 4,46 & 1,14 & 0,010 & 13,61 \\
\hline Coef. Determ. y Error Estim. & 0,523 & 2,75 & & \\
\hline \multicolumn{5}{|c|}{ cortante paralelo EMAX (Mpa) } \\
\hline & No./5mm & Altura & Anchura & $\mathrm{B}$ \\
\hline Coeficientes & $-2,64$ & 0,28 & $-0,002$ & 15,47 \\
\hline Error estándar & 1,19 & 0,31 & 0,003 & 3,64 \\
\hline Coef. Determ. y Error Estim. & 0,784 & 0,74 & & \\
\hline
\end{tabular}




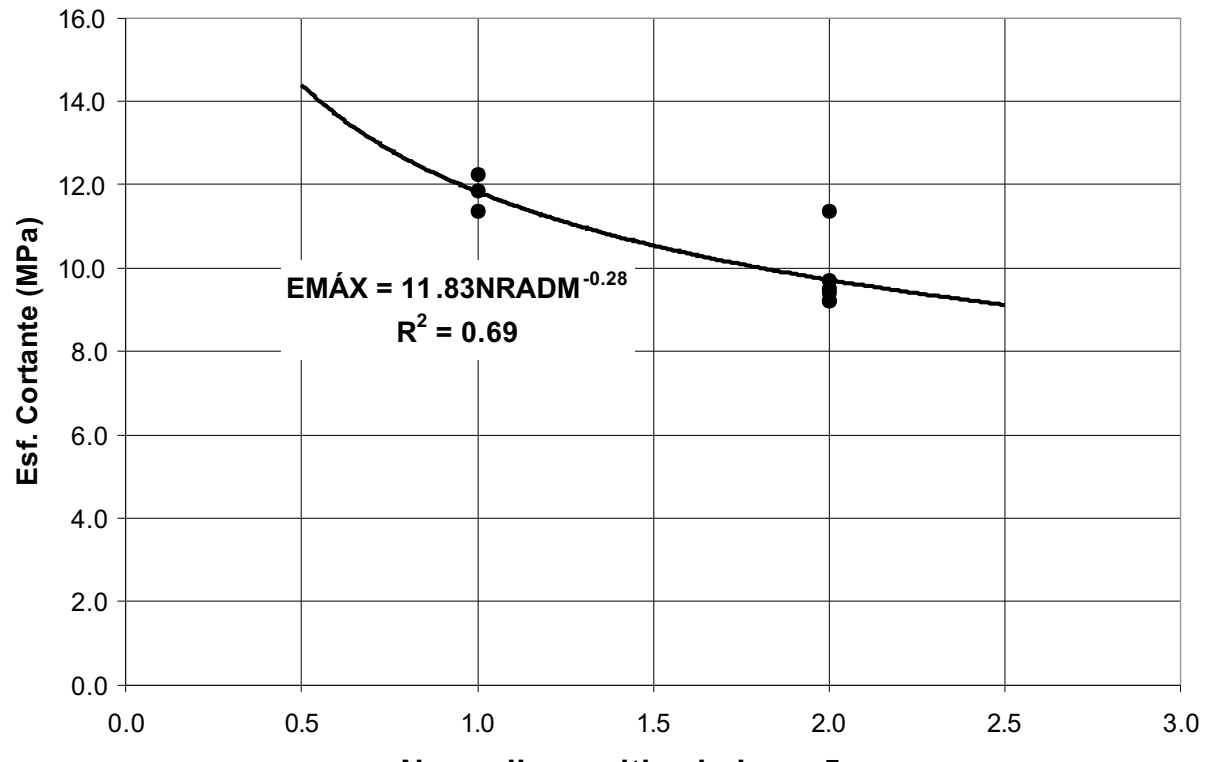

No. radios multiseriados $\times 5 \mathrm{~mm}$

Fig. 6 Curva de regresión de los radios multiseriados por $5 \mathrm{~mm}$ y el cortante paralelo EMAX

Tabla 7. Relación de las propiedades físicas y mecánicas con los radios

\begin{tabular}{|c|c|c|}
\hline \multirow[t]{2}{*}{ PROPIEDAD } & \multicolumn{2}{|c|}{ RADIOS } \\
\hline & UNISERIADOS & MULTISERIADOS \\
\hline contracción volumétrica \% & & anchura y número de series \\
\hline flexión MOR y MOE & & anchura y altura \\
\hline Compresión paralela EMAX & & altura \\
\hline compresión perpendicular ELP & número por mm & \\
\hline cortante paralelo EMAX & & altura y número por $5 \mathrm{~mm}$ \\
\hline
\end{tabular}

el segundo renglón se indican los errores estadísticos de cada uno de estos coeficientes. Finalmente en el tercer renglón se señala el coeficiente de determinación $\left(R^{2}\right)$ en primera instancia y el error estándar de la estimación.

Para determinar la relación entre el parénquima radial y las propiedades tecnológicas estudiadas, se tomaron en cuenta únicamente las variables anatómicas que se refieren a los radios (Tabla 6), mostrando, que las características de los radios multiseriados tienen gran influencia en la mayoría de las propiedades tecnológicas, principalmente su anchura y su altura. Por otro lado el número de series es importante en la contracción y el número de radios uniseriados por milímetro es esencial en la compresión perpendicular. 
Resulta interesante e ilustrativo comparar los coeficiente de determinación obtenidos en este estudio (Tabla 6) con los presentados por de la Paz Pérez (2000) (Tabla 5). La diferencia encontrada es pequeña excepto en los casos de la compresión paralela a la fibra (EMAX) y la compresión perpendicular (ELP) para las cuales, la influencia de los radios resulta menor a la que presentan las fibras. El hecho de que los valores de $R^{2}$ resulten casi iguales para la contracción volumétrica, el módulo de ruptura y el módulo de elasticidad en flexión y la resistencia en el cortante paralelo indica que se puede obtener un grado semejante de precisión en la predicción de los valores de las características tecnológicas estudiadas midiendo únicamente las variables correspondientes a los radios. Por otro lado, para la compresión paralela y perpendicular, resulta imprescindible medir las características de las fibras.

En el caso de la madera de encino, además de las dimensiones de los radios multiseriados, debe tenerse especial interés por las dimensiones de las fibras y tipo y abundancia de los contenidos celulares (tílides y cristales), que favorecen o complican sus procesos de transformación. La anchura de los anillos de crecimiento de las especies con porosidad anular, constituye un criterio valioso para juzgar la calidad de la madera (Dinwoodie, 1975; Wangaard, 1981; de la Paz Pérez et al., 1997).

Los encinos, a pesar de ser el recurso maderable más abundante de las latifoliadas en México, con alrededor de 105 especies (Aguilar et al., 1999), sufren el problema de que en los procesos de utilización de las especies de pino, con las que generalmente forman los bosques de clima templado, se talan indiscriminada e injustificadamente, ocasionando un alto desperdicio de este valioso recurso y un lamentable deterioro ecológico. En la actualidad, México importa gran cantidad de productos de madera de encino de Norteamérica y Canadá (de la Paz Pérez et al., 2000), desaprovechando el potencial que la mayoría de sus especies ofrece.

Dado el número de especies de encinos que vegetan en México y su amplia distribución (Zavala, 1995; Aguilar et al., 1999), es urgente una selección de las que presenten mejores características tecnológicas según lo que se quiera producir y hacer silvicultura con ellas. Es urgente la necesidad de fomentar programas de conservación, manejo y aprovechamiento de esta importante fuente de materias primas, así como el conocimiento del equilibrio natural de los ecosistemas donde crecen. Es necesario recordar que la explotación silvícola no debe ni puede estar basada en la tala despiadada ni en las vedas totales; la riqueza forestal de un país debe explotarse racional e íntegramente en beneficio del propio sistema ecológico y de la economía nacional.

\section{CONCLUSIONES Y SUGERENCIAS}

1. Los encinos blancos estudiados presentaron valores anatómicos y tecnológicos más altos que los encinos rojos.

2. Las características anatómicas de los radios que mayor influencia tuvieron en las propiedades tecnológicas fueron la anchura y la altura.

3. La anchura y la altura son importantes en la flexión.

4. La anchura y el número de series son importantes en la contracción

5. La altura y el número de radios en 5 
mm son importantes en el cortante.

6. La altura por si sóla tiene influencia en la compresión paralela.

7. El número de radios uniseriados por milímetro por si sólos tiene influencia en la compresión perpendicular.

En los procesos de transformación se sugiere:

1. Hacer una separación de especies por subgénero .

2. Seleccionar a las especies por las dimensiones de los radios multiseriados.

3. Establecer criterios de usos de acuerdo con sus propiedades.

\section{AGRADECIMIENTOS}

Los autores agradecen a la M. en C. Irma Reyes Jaramillo y a la Arq. Guadalupe Bárcenas Pazos. sus valiosos comentarios y sugerencias que enriquecieron el trabajo, al Mtro. Jorge Lodigiani Rodríguez, el procesado del material fotográfico y al Biól. Miguel Bravo Rivera la elaboración de la figura 1.

\section{BIBLIOGRAFÍA}

Aguilar, L., C., de la Paz Pérez O. y E. Guerrero C. 1999. Árboles y arbustos del género Quercus, especies y distribución. IV Congreso Mexicano sobre Recursos Forestales. Universidad Juárez del Estado de Durango. Durango, Dgo.

ASTM. 1993. Standard methods of testing small clear specimens of timber. ASTM Standard D-143-83. Philadelphia. PA.

Ávila S., C. G. 1985. Secado en estufa de la madera aserrada de mezcla de encinos blancos y rojos. II Seminario Nacional
Sobre Utilización de Encinos. Pub. Esp. Inst. Nac. Invest. For. No. 49:218-228.

Chattaway, M. 1932. Proposed standars for numerical values used in describing woods. Trop. Woods 9:20-28

Dávalos S., R y G. Bárcenas P.1998. Clasificación de las propiedades mecánicas en condición verde. Madera y Bosques 4(2):81-86.

De la Paz Pérez O., C. 1974. Anatomía de la madera de cinco especies de encinos de Durango. Bol. Téc. Inst. Nac. Invest. For No. 43. 32p.

De la Paz Pérez O., C. \& L. Aguilar E. 1978. Diferencias morfológicas externas y anatómicas de la madera de los encinos blancos y rojos. Bol. Téc. Inst. Nac. Invest. For. No. 59. 19p.

De la Paz Pérez O., C. y R. Dávalos. 1995. Relación estructura propiedades de la madera de dos especies de Quercus. III Seminario Nacional Sobre Utilización de Encinos. Universidad Autónoma de Nuevo León II:427-441.

De la Paz Pérez O., C., R. Dávalos S. y A. Quintanar I. 1997. Las características tecnológicas de la madera. ContactoS 19:15-21.

De la Paz Pérez O., C., R. Dávalos S. y E. Guerrero C. 2000. Aprovechamiento de la madera de encino en México. Madera y Bosques 1:3-13.

De la Paz Pérez O., C. 2000. Relación estructura propiedades fisicomecánicas de la madera de algunas especies de encinos (Quercus) 
mexicanas. Tesis Doctoral. Facultad de Ciencias. UNAM. 266p.

Desch, H. E. 1974. Timber, its structure and properties. Macmillan. 424p.

Dinwoodie, J. H. 1975. Timber, a review of the structure, mechanical properties, relationships. I.104(1):3-32.

Echenique, R., J. Barajas, L. Pinzón y V. Pérez. 1975. Estudio botánico y ecológico de la región del rio Uxpanapa, Ver. 1. Características tecnológicas de la madera de 10 especies. INIREB. 66p.

Echenique, R y F. Robles. 1993. Ciencia y tecnología de la madera I. Textos Universitarios. Universidad Veracruzana. México. 137p.

Guerrero, L., A. Guzzi, G. Bárcenas y F. Ortega. 1995. Relación de la estructura de la madera de Quercus sartorii Liebm. con cuatro propiedades físicomecánicas. III Seminario Nacional Sobre Utilización de Encinos. Universidad Autónoma de Nuevo León II:475-496.

IAWA Committee. 1939. Standard terms of size for vessel diameter and ray width. Tropical Woods 59:51-52.

IAWA Committee. 1989. IAWA list of microscopic features for hardwood identification. IAWA Bull.10(3):219-332.

Jane, J. W. 1970. The structure of wood. Adam \& Charles Black. Londres. 478p.

Kollman, F. P. y W. A. Coté, Jr. 1968. Principles of wood science and technology I. Solid Wood. SpringerVerlag Nueva York.560 p.
Lindsay, F. W. y L. Chalk. 1954. The influence of rays on the shrinkage of wood. Forestry 27:16-24.

Maeglin, R. y J. Quirk. 1984. Tissue proportions and cell dimensions for red and white oak groups. Forest Products Laboratories. Can. J. Res. 14:101-106.

McKintosh, D. C. 1954. Some aspects of the influence of rays on the shrinkage of wood. J. For. Prod. Res. Sc. 4:39-42.

Montes R., E. 1985. Alternativas para el secado de la madera de encino. II Seminario Nacional Sobre Utilización de Encinos. Pub. Esp. Inst. Nac. Invest. For. No. 49:229-237.

Negrete, J. 1970. Algunas características físicas y anatómicas de la madera de cuatro especies de encinos (Quercus) del estado de Michoacán. Tesis. Escuela Nacional de Agricultura. México. 65p.

Panshin, A. J. y C. de Zeeuw. 1970. Textbook of wood technology. I. McGraw-Hill. Nueva York. 705p.

SPP. 1996. Anuario estadístico del estado de Durango. INEGI-Gobierno del estado de Durango. 366p.

Tsoumis, G. 1969. Wood as a raw material. Pergamon Press. 276p.

Wangaard, F. F. 1981. Wood: its structure and properties. I. Forest Products Laboratory. USA. 465p.

Zavala Ch., F. 1995. Encinos y robles, notas fitogeográficas. División de Ciencias Forestales. Universidad Autónoma Chapingo. México. 44p. 
Anexo 1. Clasificación de los valores anatómicos, físicos y mecánicos

\section{RADIOS}

NÚMERO POR MM (Chattaway 1932)

$<2$

$2-4$

$5-7$

$8-10$

muy pocos

pocos

moderadamente numerosos

$>10$

numerosos

muy numerosos

ALTURA(Chattaway 1932)

$<0,5 \mathrm{~mm}$

$0,5 \mathrm{~mm}-1,0 \mathrm{~mm}$

$1,01 \mathrm{~mm}-2,0 \mathrm{~mm}$

extremadamente bajos

$2,01 \mathrm{~mm}-5,0 \mathrm{~mm}$

muy bajos

bajos

algo bajos

$5,01 \mathrm{~mm}-1,0 \mathrm{~cm}$ moderadamente altos

$1,01 \mathrm{~mm}-2,0 \mathrm{~cm}$ altos

$2,01 \mathrm{~mm}-5,0 \mathrm{~cm}$ muy altos

$>5,0 \mathrm{~cm} \quad$ extremadamente altos

ANCHURA (IAWA 1939)

$<15 \mu$

$15 \mu-25 \mu$

$26 \mu-50 \mu$

$51 \mu-100 \mu$

$101 \mu-200 \mu$

$201 \mu-400 \mu$

$>400 \mu$

extremadamente finos

muy finos

moderadamente finos

medianos

moderadamente anchos

muy anchos

extremadamente anchos

CONTRACCIÓN VOLUMÉTRICA (\%) (Echenique et al 1975)

$10,1-15$

$15,1-19$

$>19$

mediana

alta

muy alta

FLEXIÓN MOR (MPa) (Dávalos y Bárcenas 1998)

(módulo de ruptura)

$28,5-50$

$50,165,0$

flexible

$65,190,0$

$>900$

moderadamente flexible

rígida

muy rígida

FLEXIÓN MOE (MPa) (Dávalos y Bárcenas 1998)

(módulo de elasticidad)

55008000

flexible

$8100-10000$

1000112500

moderadamente flexible

rígida 
$>12500 \quad$ muy rígida

COMPRESIÓN PARALELA EMAX (MPa)(Dávalos y Bárcenas 1998)

(esfuerzo máximo)

$16,125,0$

$25,131,0$

poco resistente

$31,1 \quad 40,0$

moderadamente resistente

$>40,0$

resistente

muy resistente

COMPRESIÓN PERPENDICULAR ELP (MPa) (Dávalos y Bárcenas 1998)

(esfuerzo al límite proporcional)

$16,125,0$

$25,131,0$

$31,1 \quad 40,0$

$>40,0$

poco resistente

moderadamente resistente

resistente

muy resistente

CORTANTE EMAX (MPa)(Dávalos y Bárcenas 1998)

(esfuerzo máximo)

$6,5 \quad 8,5$

$8,6 \quad 11,0$

$>11,0$

moderadamente resistente

resistente

muy resistente

Manuscrito recibido el 14 de mayo de 2005

Aceptado el 7 de octubre de 2005

Este documento se debe citar como:

de la Paz Pérez, C., R. Dávalos S. y P. A. Quintanar. 2005. Influencia de los radios en algunas propiedades físicas y mecánicas de la madera de ocho encinos (Quercus) de Durango, México. Madera y Bosques 11(2):49-68 